\title{
Article \\ In Vitro Efficacy of Flomoxef against Extended-Spectrum Beta-Lactamase-Producing Escherichia coli and Klebsiella pneumoniae Associated with Urinary Tract Infections in Malaysia
}

\author{
Soo Tein Ngoi ${ }^{1}{ }^{(0)}$, Cindy Shuan Ju Teh ${ }^{1, *}$, Chun Wie Chong ${ }^{2}$, Kartini Abdul Jabar ${ }^{1}{ }^{\circledR}$, Shiang Chiet Tan ${ }^{1}$, \\ Lean Huat $\mathbf{Y u}^{1}$, Kin Chong Leong ${ }^{3}$, Loong Hua Tee ${ }^{3}$ and Sazaly AbuBakar ${ }^{1,4}$ \\ 1 Department of Medical Microbiology, Faculty of Medicine, University of Malaya, Kuala Lumpur 50603, \\ Malaysia; ngoisootein@um.edu.my (S.T.N.); kartini.abduljabar@ummc.edu.my (K.A.J.); \\ tanshiangchiet@gmail.com (S.C.T.); leoyu_1993@hotmail.com (L.H.Y.); sazaly@um.edu.my (S.A.) \\ 2 School of Pharmacy, Monash University Malaysia, Bandar Sunway 47500, Selangor, Malaysia; \\ chong.chunwie@monash.edu \\ 3 Shionogi Singapore Pte Ltd., 10, Anson Road, \#34-14 International Plaza, Singapore 079903, Singapore; \\ kinchong.leong@shionogi.com.sg (K.C.L.); loonghua.tee@shionogi.com.sg (L.H.T.) \\ 4 Tropical Infectious Diseases Research and Education Centre (TIDREC), University of Malaya, \\ Kuala Lumpur 50603, Malaysia \\ * Correspondence: cindysjteh@um.edu.my; Tel.: +60-379676674
}

Citation: Ngoi, S.T.; Teh, C.S.J.; Chong, C.W.; Abdul Jabar, K.; Tan, S.C.; Yu, L.H.; Leong, K.C.; Tee, L.H.; AbuBakar, S. In Vitro Efficacy of Flomoxef against Extended-Spectrum Beta-Lactamase-Producing Escherichia coli and Klebsiella pneumoniae Associated with Urinary Tract Infections in Malaysia. Antibiotics 2021, 10, 181. https://doi.org/ 10.3390/antibiotics10020181

Academic Editor:

Ivo Gomperts-Boneca

Received: 7 January 2021

Accepted: 4 February 2021

Published: 11 February 2021

Publisher's Note: MDPI stays neutral with regard to jurisdictional claims in published maps and institutional affiliations.

Copyright: () 2021 by the authors. Licensee MDPI, Basel, Switzerland. This article is an open access article distributed under the terms and conditions of the Creative Commons Attribution (CC BY) license (https:// creativecommons.org/licenses/by/ $4.0 /)$.

\begin{abstract}
The increasing prevalence of extended-spectrum $\beta$-lactamase (ESBL)-producing Enterobacteriaceae has greatly affected the clinical efficacy of $\beta$-lactam antibiotics in the management of urinary tract infections (UTIs). The limited treatment options have resulted in the increased use of carbapenem. However, flomoxef could be a potential carbapenem-sparing strategy for UTIs caused by ESBL-producers. Here, we compared the in vitro susceptibility of UTI-associated ESBL-producers to flomoxef and established $\beta$-lactam antibiotics. Fifty Escherichia coli and Klebsiella pneumoniae strains isolated from urine samples were subjected to broth microdilution assay, and the presence of ESBL genes was detected by polymerase chain reactions. High rates of resistance to amoxicillin-clavulanate (76-80\%), ticarcillin-clavulanate (58-76\%), and piperacillin-tazobactam (48-50\%) were observed, indicated by high minimum inhibitory concentration (MIC) values ( $32 \mu \mathrm{g} / \mathrm{mL}$ to $128 \mu \mathrm{g} / \mathrm{mL}$ ) for both species. The ESBL genes $b l a_{\mathrm{CTX}-\mathrm{M}}$ and $b l a_{\mathrm{TEM}}$ were detected in both $E$. coli ( $58 \%$ and $54 \%$, respectively) and K. pneumoniae ( $88 \%$ and $74 \%$, respectively), whereas $b l a_{\mathrm{SHV}}$ was found only in K. pneumoniae (94\%). Carbapenems remained as the most effective antibiotics against ESBL-producing E. coli and K. pneumoniae associated with UTIs, followed by flomoxef and cephamycins. In conclusion, flomoxef may be a potential alternative to carbapenem for UTIs caused by ESBL-producers in Malaysia.
\end{abstract}

Keywords: antimicrobial resistance; $\beta$-lactamase inhibitor; broad-spectrum $\beta$-lactamase; resistance gene; Enterobacteriaceae

\section{Introduction}

Urinary tract infections (UTIs) include infections that affect the urethra (urethritis), urinary bladder (cystitis), or kidneys (pyelonephritis) [1]. UTIs are more prevalent in female subjects, even among individuals with a high risk of infections (e.g., catheterized patients) [2]. Based on disease classification by Infectious Diseases Society of America (IDSA) and National Antimicrobial Guidelines (NAG) Malaysia, uncomplicated UTIs include acute, symptomatic bacterial cystitis and acute pyelonephritis in nonpregnant, premenopausal women without urological abnormalities or comorbidities [3,4]. On the other hand, UTI symptoms in men or the presence of a structural or functional abnormality in the urinary tract in women are considered complicated UTIs [4]. Commonly identified etiologic 
microorganisms in UTIs include Escherichia coli, Staphylococcus spp., Klebsiella pneumoniae, Enterobacter spp., Proteus mirabilis, Enterococcus faecalis, group B Streptococcus, Pseudomonas aeruginosa, and Candida spp. [5,6]. Uncomplicated UTIs in otherwise healthy women in the community are mainly caused by uropathogenic E. coli (80\%) [2]. The second most common causative agent of UTIs is K. pneumoniae, a highly relevant bacteria in complicated UTIs where patients are predisposed to infections due to underlying healthcare-associated risk factors $[6,7]$.

Once diagnosed with UTIs, finite courses of antibiotics are often prescribed to resolve acute symptoms. The recommended first-line antibiotics for the treatment of acute uncomplicated UTIs include nitrofurantoin, trimethoprim-sulfamethoxazole, fosfomycin trometamol, and pivmecillinam (in regions where it is available), in a treatment course ranging from one to seven days $[3,8]$. In cases of treatment failure, second-line antibiotics that include fluoroquinolones and $\beta$-lactams may be prescribed for three to seven days [8]. Besides, fluoroquinolones and $\beta$-lactam antibiotics are recommended for the treatment of acute pyelonephritis and complicated UTIs [3,4]. Given the recurrent nature of UTIs, antibiotic treatments may lead to development of resistance in bacterial pathogens, compromising the effectiveness of successive treatments [2]. Moreover, the wide use of antimicrobial prophylaxis in patients predisposed to UTIs has also contributed to the rise in antimicrobial resistance (AMR) [6,7].

The increasing prevalence of AMR among the aetiologic agents of UTIs has been observed since the early $2000 \mathrm{~s}$ [5]. Worldwide emergence and prevalence of extendedspectrum $\beta$-lactamase (ESBL)-producing Enterobacteriaceae, including E. coli and K. pneumoniae, have become a serious threat to public health [9]. The Institute of Medical Research (IMR), a Ministry of Health (MOH) agency in Malaysia, has been actively monitoring AMR rates in clinical isolates since 2003. To date, 41 hospitals and one public health laboratory have contributed to the national AMR database. AMR surveillance for urine isolates has documented relatively high rates of resistance to $\beta$-lactam antibiotics such as ampicillin, amoxicillin-clavulanate, piperacillin-tazobactam, cefepime, cefotaxime, ceftazidime, and cefuroxime (10-68\% in E. coli and 5-40\% in K. pneumoniae) and low rates for carbapenem resistance (less than $1 \%$ in E. coli and less than $5 \%$ in K. pneumoniae) over the past decade [10]. ESBL-producing E. coli and K. pneumoniae have emerged and spread in Malaysia [11].

Flomoxef is a broad-spectrum oxacephem antibiotic that was introduced to the medical field in the mid-1980s [12]. The in vitro and clinical efficacy of flomoxef have been proven satisfactory in the treatment of infections caused by both Gram-positive and Gram-negative bacteria, with minimal side effects or unexpected laboratory results [13-15]. The use of flomoxef in empiric and definitive therapy has been previously evaluated and was proven to be effective for ESBL-producing E. coli bacteremia [16]. Preclinical studies in China, Japan, Korea, and Taiwan have reported that flomoxef susceptibility among ESBL-producing Enterobacteriaceae, especially in E. coli, was comparable to cefmetazole, cefoxitin, imipenem, and meropenem [17-21]. Clinical studies conducted in pediatric patients with UTIs caused by ESBL-producing E. coli showed comparable susceptibility between flomoxef, cefmetazole, and imipenem [22,23].

The in vitro activity of flomoxef on ESBL-producing Enterobacteriaceae beyond Northeast Asian region has not been investigated. The current literature showed that flomoxef may be a potential alternative to carbapenems for the treatment of UTIs caused by ESBLproducers. Hence, we aimed to investigate the in vitro efficacy of flomoxef in comparison with other established $\beta$-lactam antibiotics against ESBL-producing E. coli and K. pneumoniae associated with UTIs in Malaysia.

\section{Results}

\subsection{Patient's Demographics and Bacterial Strain Antimicrobial Susceptibility}

Approximately half of the UTI-associated ESBL-producing E. coli $(n=28 ; 56 \%)$ and $K$. pneumoniae ( $n=25 ; 50 \%$ ) strains occurred in elderly patients (aged 60 and above). Thirtytwo percent of E. coli and 36\% of K. pneumoniae strains were isolated from adult patients 
aged between 18 and 59 years old. The remaining E. coli (12\%) and K. pneumoniae (14\%) strains were isolated from patients aged less than 18 years old. Sixty-eight percent of E. coli and $52 \%$ of K. pneumoniae strains were isolated from female patients. AMR data of the bacterial strains for cefuroxime, nitrofurantoin, trimethoprim-sulfamethoxazole, and ciprofloxacin were obtained from hospital diagnostic laboratory and summarized in Table 1.

Table 1. Antimicrobial resistance data obtained from the University Malaya Medical Centre (UMMC) diagnostic laboratory database.

\begin{tabular}{ccc}
\hline Antibiotic & E. coli $(n)(\%)$ & K. pneumoniae * $(n)(\%)$ \\
\hline Cefuroxime & $50(100)$ & $49(98)$ \\
Ciprofloxacin & $35(70)$ & $20(40)$ \\
Nitrofurantoin & $1(2)$ & $20(40)$ \\
Trimethoprim- & $38(76)$ & $20(45)$ \\
sulfamethoxazole &
\end{tabular}

* Nitrofurantoin resistance data for Klebsiella pneumoniae was only available for 44 strains. E. coli: Escherichia coli.

\section{2. $\beta$-Lactam Resistance in E. coli}

Table 2 summarizes both the minimum inhibitory concentration (MIC) value and the corresponding phenotype of the ESBL-producing E. coli strains in this study. The MIC range, median $\left(\mathrm{MIC}_{50}\right)$, and $90 \%$ efficacy $\left(\mathrm{MIC}_{90}\right)$ values for all tested $\beta$-lactam antibiotics are also shown. Briefly, all E. coli strains $(n=50)$ were confirmed as ESBL-producers based on the Clinical and Laboratory Standards Institute (CLSI) guidelines. High MIC values $\left(\mathrm{MIC}_{50} \geq 64 \mu \mathrm{g} / \mathrm{mL}\right.$ ) were recorded for penicillins, third- and fourth-generation cephalosporins, and two out of five of the $\beta$-lactam combination agents when tested against ESBL-producing E. coli strains (Table 2). Carbapenems and flomoxef showed the lowest $\mathrm{MIC}_{50}$ values $(\leq 0.5 \mu \mathrm{g} / \mathrm{mL})$. In addition, most of the E. coli strains $(\mathrm{n}=41 ; 82 \%)$ showed simultaneous resistance to at least $10 \beta$-lactam antibiotics (Table 3).

Table 2. Summary of the minimum inhibitory concentration (MIC) data and susceptibility phenotypes of extended-spectrum $\beta$-lactamase (ESBL)-producing E. coli strains $(n=50)$.

\begin{tabular}{|c|c|c|c|c|c|c|}
\hline \multirow{2}{*}{ Antimicrobial Agent } & \multirow{2}{*}{$\begin{array}{c}\text { MIC Range } \\
(\mu \mathrm{g} / \mathrm{mL})\end{array}$} & \multirow{2}{*}{$\begin{array}{l}\mathrm{MIC}_{50} \\
(\mu \mathrm{g} / \mathrm{mL})\end{array}$} & \multirow{2}{*}{$\begin{array}{c}\mathrm{MIC}_{90} \\
(\mu \mathrm{g} / \mathrm{mL})\end{array}$} & \multicolumn{3}{|c|}{ Susceptibility Phenotype ${ }^{\text {a }}(n)(\%)$} \\
\hline & & & & $\mathbf{S}$ & I & $\mathbf{R}$ \\
\hline Ampicillin & $128->256$ & $>256$ & $>256$ & $0(0)$ & $0(0)$ & $50(100)$ \\
\hline Penicillin-G & $>64$ & $>64$ & $>64$ & $0(0)$ & $0(0)$ & $50(100)$ \\
\hline Piperacillin & $\geq 128$ & $>128$ & $>128$ & $0(0)$ & $0(0)$ & $50(100)$ \\
\hline Ticarcillin & $>128$ & $>128$ & $>128$ & $0(0)$ & $0(0)$ & $50(100)$ \\
\hline Amoxicillin-clavulanate & $8->64$ & 32 & $>64$ & $6(12)$ & $6(12)$ & $38(76)$ \\
\hline Ceftazidime-clavulanate & $1-128$ & 8 & 32 & NA & NA & NA \\
\hline Cefotaxime-clavulanate & $\leq 0.125-64$ & 8 & 32 & NA & NA & NA \\
\hline Ticarcillin-clavulanate & $8->128$ & 128 & $>128$ & $2(4)$ & $19(38)$ & $29(58)$ \\
\hline Piperacillin-tazobactam & $4->128$ & 64 & $>128$ & $4(8)$ & $22(44)$ & $24(48)$ \\
\hline Cefmetazole & $1->64$ & 4 & 64 & $43(86)$ & $1(2)$ & $6(12)$ \\
\hline Cefoxitin & $2->64$ & 8 & $>64$ & $29(58)$ & $10(20)$ & $11(12)$ \\
\hline Ceftazidime & $8->256$ & 64 & 256 & $0(0)$ & $1(2)$ & $49(98)$ \\
\hline Cefoperazone & $\geq 64$ & $>64$ & $>64$ & $0(0)$ & $0(0)$ & $50(100)$ \\
\hline Ceftriaxone & $32->64$ & $>64$ & $>64$ & $0(0)$ & $0(0)$ & $50(100)$ \\
\hline Cefotaxime & $4->256$ & 256 & $>256$ & $0(0)$ & $0(0)$ & $50(100)$ \\
\hline Cefepime & $\leq 0.125->256$ & $>256$ & $>256$ & $4(8)$ & $2(4)$ & $44(88)$ \\
\hline Imipenem & $\leq 0.03-0.25$ & $\leq 0.03$ & 0.06 & $50(100)$ & $0(0)$ & $0(0)$ \\
\hline Meropenem & $\leq 0.03-0.125$ & 0.06 & 0.125 & $50(100)$ & $0(0)$ & $0(0)$ \\
\hline Flomoxef $b$ & $\leq 0.125->64$ & 0.5 & 32 & $44(88)$ & $5(10)$ & $1(2)$ \\
\hline
\end{tabular}

a S: susceptible, I: intermediate, R: resistant, and NA: not available (MIC breakpoints are not provided in the Clinical and Laboratory Standards Institute (CLSI) guidelines). ${ }^{\mathrm{b}}$ Susceptibility phenotypes predicted based on MIC breakpoints for moxalactam (CLSI). 
Table 3. Antimicrobial resistance profiles of E. coli $(n=50)$.

\begin{tabular}{lc}
\hline \multicolumn{1}{c}{ AMR Profiles * } & No. (\%) of Strains \\
\hline AMP, PCG, PIP, TIC, AMC, TIM, TZP, CMZ, FOX, CAZ, CFP, CRO, CTX, FEP & $3(6)$ \\
AMP, PCG, PIP, TIC, AMC, TIM, TZP, FOX, CAZ, CFP, CRO, CTX, FEP & $2(4)$ \\
AMP, PCG, PIP, TIC, AMC, TZP, CMZ, FOX, CAZ, CFP, CRO, CTX, FEP & $1(2)$ \\
AMP, PCG, PIP, TIC, AMC, TIM, CMZ, FOX, CAZ, CFP, CRO, CTX & $1(2)$ \\
AMP, PCG, PIP, TIC, AMC, TIM, FOX, CAZ, CFP, CRO, CTX, FEP & $2(4)$ \\
AMP, PCG, PIP, TIC, AMC, TIM, TZP, CAZ, CFP, CRO, CTX, FEP & $13(26)$ \\
AMP, PCG, PIP, TIC, AMC, TIM, TZP, FOX, CAZ, CFP, CRO, CTX & $1(2)$ \\
AMP, PCG, PIP, TIC, AMC, CMZ, FOX, CAZ, CFP, CRO, CTX & $1(2)$ \\
AMP, PCG, PIP, TIC, AMC, TIM, CAZ, CFP, CRO, CTX, FEP & $5(10)$ \\
AMP, PCG, PIP, TIC, AMC, TZP, CAZ, CFP, CRO, CTX, FEP & $1(2)$ \\
AMP, PCG, PIP, TIC, AMC, CAZ, CFP, CRO, CTX, FEP & $6(12)$ \\
AMP, PCG, PIP, TIC, AMC, TIM, CAZ, CFP, CRO, CTX & $1(2)$ \\
AMP, PCG, PIP, TIC, TIM, CAZ, CFP, CRO, CTX, FEP & $1(2)$ \\
AMP, PCG, PIP, TIC, TZP, CAZ, CFP, CRO, CTX, FEP & $3(6)$ \\
AMP, PCG, PIP, TIC, AMC, CAZ, CFP, CRO, CTX & $1(2)$ \\
AMP, PCG, PIP, TIC, CAZ, CFP, CRO, CTX, FEP & $7(14)$ \\
AMP, PCG, PIP, TIC, CFP, CRO, CTX & $1(2)$ \\
\hline
\end{tabular}

* AMP: ampicillin, PCG: penicillin-G, PIP: piperacillin, TIC: ticarcillin, AMC: amoxicillin-clavulanate, TIM: ticarcillin-clavulanate, TZP: piperacillin-tazobactam, CMZ: cefmetazole, FOX: cefoxitin, CAZ: ceftazidime, CFP: cefoperazone, CRO: ceftriaxone, CTX: cefotaxime, and FEP: cefepime.

\section{3. $\beta$-Lactam Resistance in K. pneumoniae}

All K. pneumoniae strains $(n=50)$ were confirmed to be ESBL-producers based on the CLSI guidelines. Similar to the E. coli strains examined in this study, high MIC values $\left(\mathrm{MIC}_{50} \geq 64 \mu \mathrm{g} / \mathrm{mL}\right.$ ) were recorded for penicillins, $\beta$-lactam combination agents (except ceftazidime-clavulanate and cefotaxime-clavulanate), and third- and fourth-generation cephalosporins (Table 4). Carbapenems and flomoxef showed the lowest MIC values $\left(\mathrm{MIC}_{50} \leq 1 \mu \mathrm{g} / \mathrm{mL}\right)$, although slightly increased compared to that of the E. coli strains. In general, K. pneumoniae strains showed comparable AMR phenotypes to E. coli. Most of the K. pneumoniae strains (90\%) were simultaneously resistant to at least $10 \beta$-lactam antibiotics (Table 5).

Table 4. Summary of the MIC data and susceptibility phenotypes of ESBL-producing K. pneumoniae strains $(n=50)$.

\begin{tabular}{|c|c|c|c|c|c|c|}
\hline \multirow{2}{*}{ Antimicrobial Agent } & \multirow{2}{*}{$\begin{array}{c}\text { MIC Range } \\
(\mu \mathrm{g} / \mathrm{mL})\end{array}$} & \multirow{2}{*}{$\begin{array}{c}\mathrm{MIC}_{50} \\
(\mu \mathrm{g} / \mathrm{mL})\end{array}$} & \multirow{2}{*}{$\begin{array}{c}\mathrm{MIC}_{90} \\
(\mu \mathrm{g} / \mathrm{mL})\end{array}$} & \multicolumn{3}{|c|}{ Susceptibility Phenotype ${ }^{a}$ (n) (\%) } \\
\hline & & & & $\mathbf{S}$ & I & $\mathbf{R}$ \\
\hline Ampicillin & $>256$ & $>256$ & $>256$ & $0(0)$ & $0(0)$ & $50(100)$ \\
\hline Penicillin-G & $>64$ & $>64$ & $>64$ & $0(0)$ & $0(0)$ & $50(100)$ \\
\hline Piperacillin & $>128$ & $>128$ & $>128$ & $0(0)$ & $0(0)$ & $50(100)$ \\
\hline Ticarcillin & $>128$ & $>128$ & $>128$ & $0(0)$ & $0(0)$ & $50(100)$ \\
\hline Amoxicillin-clavulanate & $4->64$ & 64 & $>64$ & $7(14)$ & $3(6)$ & $40(80)$ \\
\hline Ceftazidime-clavulanate & $2-256$ & 16 & 64 & NA & NA & NA \\
\hline Cefotaxime-clavulanate & $0.25-256$ & 16 & 64 & NA & NA & NA \\
\hline Ticarcillin-clavulanate & $32->128$ & 128 & $>128$ & $0(0)$ & $12(24)$ & $38(76)$ \\
\hline Piperacillin-tazobactam & $4->128$ & 64 & $>128$ & $8(16)$ & $17(34)$ & $25(50)$ \\
\hline Cefmetazole & $0.25->64$ & 2 & 16 & $45(90)$ & $2(4)$ & $3(6)$ \\
\hline Cefoxitin & $2->64$ & 8 & 32 & $31(62)$ & $9(18)$ & $10(20)$ \\
\hline Ceftazidime & $8->256$ & 64 & 256 & $0(0)$ & $4(8)$ & $46(92)$ \\
\hline Cefoperazone & $\geq 64$ & $>64$ & $>64$ & $0(0)$ & $0(0)$ & $50(100)$ \\
\hline Ceftriaxone & $>64$ & $>64$ & $>64$ & $0(0)$ & $0(0)$ & $50(100)$ \\
\hline Cefotaxime & $32->256$ & $>256$ & $>256$ & $0(0)$ & $0(0)$ & $50(100)$ \\
\hline Cefepime & $64->256$ & $>256$ & $>256$ & $0(0)$ & $0(0)$ & $50(100)$ \\
\hline Imipenem & 0.25-1 & 1 & 1 & $50(100)$ & $0(0)$ & $0(0)$ \\
\hline Meropenem & $<0.03-0.5$ & 0.06 & 0.125 & $50(100)$ & $0(0)$ & $0(0)$ \\
\hline Flomoxef ${ }^{b}$ & $0.06->64$ & 1 & 8 & $45(90)$ & $3(6)$ & $2(4)$ \\
\hline
\end{tabular}

\footnotetext{
a S: susceptible, I: intermediate, R: resistant, and NA: not available (MIC breakpoints are not provided in the CLSI guidelines).
}

b Susceptibility phenotypes predicted based on the MIC breakpoints for moxalactam (CLSI). 
Table 5. Antimicrobial resistance profiles of K. pneumoniae $(n=50)$.

\begin{tabular}{lc}
\hline \multicolumn{1}{c}{ AMR Profile * } & No. (\%) of Strains \\
\hline AMP, PCG, PIP, TIC, AMC, TIM, CMZ, FOX, CAZ, CFP, CRO, CTX, FEP & $1(2)$ \\
AMP, PCG, PIP, TIC, AMC, TIM, TZP, FOX, CAZ, CFP, CRO, CTX, FEP & $4(8)$ \\
AMP, PCG, PIP, TIC, AMC, TIM, FOX, CAZ, CFP, CRO, CTX, FEP & $1(2)$ \\
AMP, PCG, PIP, TIC, AMC, TIM, TZP, CAZ, CFP, CRO, CTX, FEP & $16(32)$ \\
AMP, PCG, PIP, TIC, AMC, TZP, FOX, CAZ, CFP, CRO, CTX, FEP & $2(4)$ \\
AMP, PCG, PIP, TIC, TIM, CMZ, FOX, CAZ, CFP, CRO, CTX, FEP & $1(2)$ \\
AMP, PCG, PIP, TIC, TIM, TZP, CMZ, FOX, CFP, CRO, CTX, FEP & $1(2)$ \\
AMP, PCG, PIP, TIC, AMC, TIM, CAZ, CFP, CRO, CTX, FEP & $8(16)$ \\
AMP, PCG, PIP, TIC, AMC, TIM, TZP, CFP, CRO, CTX, FEP & $1(2)$ \\
AMP, PCG, PIP, TIC, TIM, TZP, CAZ, CFP, CRO, CTX, FEP & $1(2)$ \\
AMP, PCG, PIP, TIC, AMC, CAZ, CFP, CRO, CTX, FEP & $5(10)$ \\
AMP, PCG, PIP, TIC, AMC, TIM, CFP, CRO, CTX, FEP & $1(2)$ \\
AMP, PCG, PIP, TIC, TIM, CAZ, CFP, CRO, CTX, FEP & $3(6)$ \\
AMP, PCG, PIP, TIC, AMC, CFP, CRO, CTX, FEP & $1(2)$ \\
AMP, PCG, PIP, TIC, CAZ, CFP, CRO, CTX, FEP & $4(8)$ \\
\hline
\end{tabular}

* AMP: ampicillin, PCG: penicillin-G, PIP: piperacillin, TIC: ticarcillin, AMC: amoxicillin-clavulanate, TIM: ticarcillin-clavulanate, TZP: piperacillin-tazobactam, CMZ: cefmetazole, FOX: cefoxitin, CAZ: ceftazidime, CFP: cefoperazone, CRO: ceftriaxone, CTX: cefotaxime, and FEP: cefepime.

\subsection{ESBL Genes Detected in E. coli and K. pneumoniae Strains}

The ESBL genes $b l a_{\mathrm{CTX}} \mathrm{M}$ and $b l a_{\mathrm{TEM}}$ were commonly detected among the UTIassociated E. coli (58\% and $54 \%$ ) and K. pneumoniae ( $88 \%$ and $74 \%$ ) strains examined in this study (Table 6). The blasHV gene was detected only in K. pneumoniae strains (94\%), while the $b l a_{\mathrm{FOX}}$ gene was absent in both the E. coli and K. pneumoniae strains. The presence of at least two ESBL genes was more commonly seen in K. pneumoniae (86\%) than in E. coli (34\%) strains.

Table 6. ESBL genes profiles of E. coli and K. pneumoniae.

\begin{tabular}{ccc}
\hline ESBL Gene Profiles & K. pneumoniae $(\boldsymbol{n}) \mathbf{( \% )}$ & E. coli $(\boldsymbol{n}) \mathbf{( \% )}$ \\
\hline bla $_{\mathrm{CTX}-\mathrm{M}}, b l a_{\mathrm{TEM}}, b l a_{\mathrm{SHV}}$ & $34(68)$ & $0(0)$ \\
bla $_{\mathrm{CTX}-\mathrm{M}}, b l a_{\mathrm{SHV}}$ & $6(12)$ & $0(0)$ \\
bla $_{\mathrm{CTX}-\mathrm{M}}, b l a_{\mathrm{TEM}}$ & $3(6)$ & $17(34)$ \\
$b l a_{\mathrm{CTX}-\mathrm{M}}$ & $1(2)$ & $12(24)$ \\
$b l a_{\mathrm{SHV}}$ & $6(6)$ & $0(0)$ \\
bla & $0(0)$ & $10(20)$ \\
None & $0(0)$ & $11(22)$ \\
\hline
\end{tabular}

\subsection{Flomoxef Activity in ESBL-Producing E. coli and K. pneumoniae}

Flomoxef effectively inhibits most of the E. coli strains (78\%) and approximately half of the K. pneumoniae strains $(56 \%)$ at lower concentrations $(\leq 1 \mu \mathrm{g} / \mathrm{mL})$. Ten percent of E. coli strains and $30 \%$ of $K$. pneumoniae strains were inhibited by a higher flomoxef concentration $(2-4 \mu \mathrm{g} / \mathrm{mL}$ ). A potentially non-susceptible phenotype (MIC $\geq 8 \mu \mathrm{g} / \mathrm{mL}$ ) to flomoxef was observed in both the E. coli (12\%) and K. pneumoniae (14\%) strains. The relative efficiency of flomoxef in comparison with imipenem, meropenem, and cefmetazole is shown in Figure 1. Simultaneous susceptibility to the selected antimicrobial agents was common in both $E$. coli and K. pneumoniae, indicated by clusters formed at $\log _{10}$ MIC $\leq 1$ in the scatter plots. Generally speaking, the MICs of carbapenems remained consistently low regardless of the increasing resistance to flomoxef. A positive relationship was inferred for the susceptibility of E. coli and K. pneumoniae to cefmetazole and flomoxef, as shown by upward trendlines in the scatter plots. 


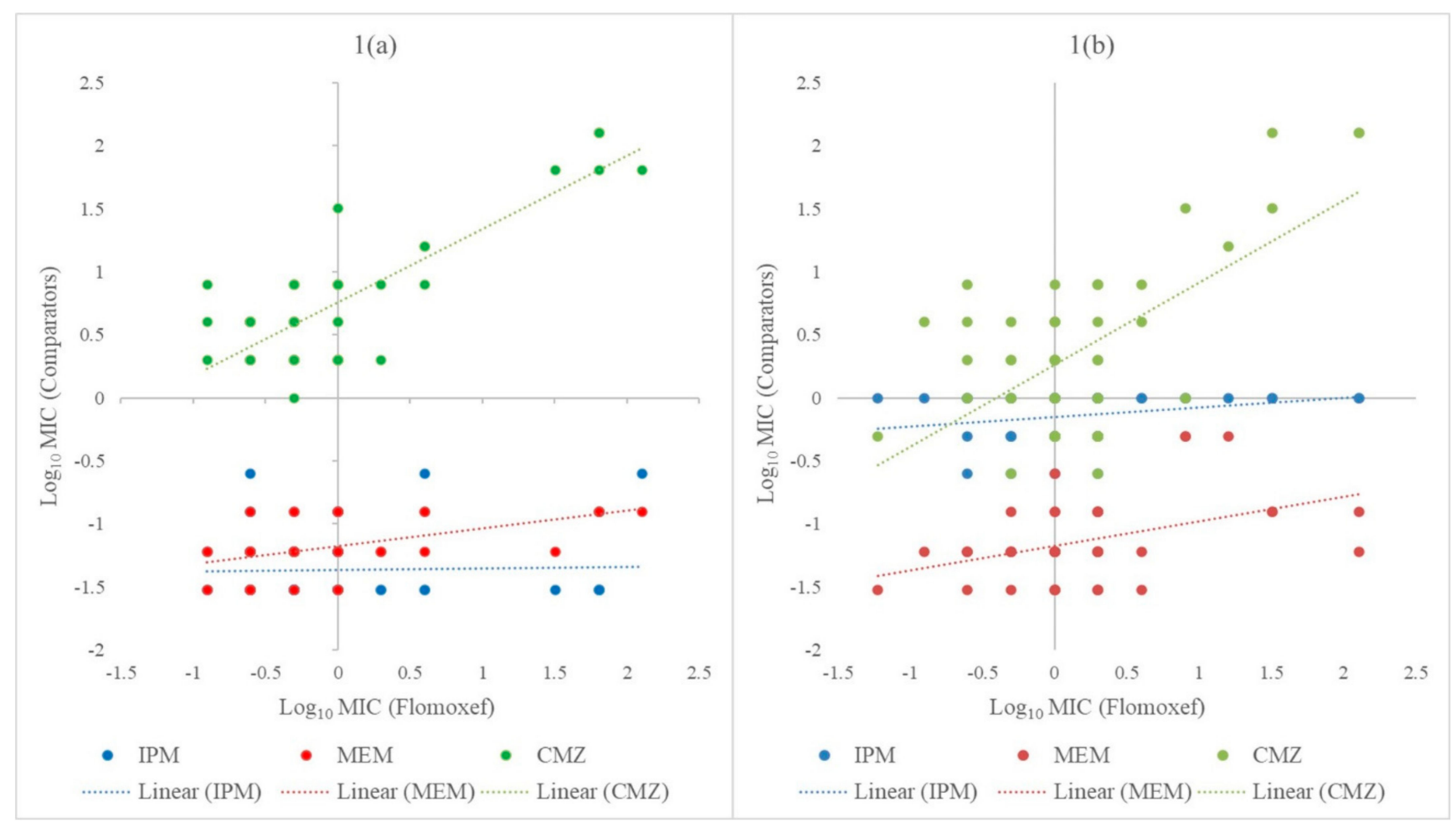

Figure 1. Scatter plots to show the relationship between the minimum inhibitory concentration (MIC) values of imipenem, meropenem, and cefmetazole against flomoxef for E. coli (1a) and K. pneumoniae (1) $)$. The dotted lines indicate the linear relationship between the comparators (imipenem, meropenem, or cefmetazole) and flomoxef. CMZ: cefmetazole, IPM: imipenem, MEM: meropenem, and MIC: minimum inhibitory concentration.

\section{Discussion}

We observed high rates of resistance to penicillin antibiotics, third- and fourthgeneration cephalosporins, and a high rate of nonsusceptibility to $\beta$-lactam combination agents among the ESBL-producing E. coli and K. pneumoniae strains isolated from urine samples. Both organisms showed similar AMR trends when tested with $\beta$-lactam antibiotics. The multi-resistance patterns detected in the E. coli and K. pneumoniae strains in this study were accompanied, in most cases, by ESBL-encoding genes. All ESBL-producing strains examined in this study were highly susceptible to carbapenems with low MIC values. Similarly, flomoxef exhibited an inhibitory effect on the ESBL producers at low concentrations, especially in E. coli.

The high rate of resistance to penicillins, cephalosporins (except cephamycins), trimeth oprim-sulfamethoxazole, and ciprofloxacin observed in this study suggested that these antibiotics might be ineffective in the treatment of UTIs caused by ESBL-producing E. coli and K. pneumoniae in this region. These antibiotics are commonly prescribed in Malaysian primary care settings, constituting up to $93 \%$ of antibiotics prescribed for UTI treatment [24]. $\beta$-lactam antibiotics, including penicillins, cephalosporins, and penicillin combinations with an enzyme inhibitor, are the most prescribed antibiotics in both public hospitals and private practice in Malaysia, accounting for approximately $66 \%$ of all antibiotics prescribed in primary care settings [25]. The overuse of these antibiotics could have contributed to the development of resistance among E. coli and K. pneumoniae strains circulating in Malaysia. The NAG recommended an empirical drug for uncomplicated cystitis, nitrofurantoin, which remained highly effective against ESBL-producing E. coli and, to a lesser extent, $K$. pneumoniae in our study [4]. Amoxicillin-clavulanate is recommended as an alternative drug for the treatment of uncomplicated cystitis and a drug of choice for pyelonephritis and complicated UTIs in Malaysia [4]. However, high rates of resistance to amoxicillinclavulanate were observed in ESBL-producing E. coli and K. pneumoniae in this study. 
Therefore, obtaining a urine sample for culture and susceptibility testing before starting treatment is essential to avoid treatment failure in infections caused by ESBL-producers.

Combining our findings with that of the national surveillance data, we observed that a greater resistance to penicillins, cephalosporins, and $\beta$-lactam combination agents is probably common among ESBL-producing E. coli and K. pneumoniae associated with UTIs, and carbapenems remained the most effective therapeutic agents in this region [10]. Comparing to in vitro studies in China and Korea, which also examined a wide array of different $\beta$-lactam antibiotics, a similar AMR trend was observed among the ESBL producers $[19,21]$. ESBL-producing E. coli and K. pneumoniae reported in these studies were generally highly resistant to cephalosporins $\left(\mathrm{MIC}_{50 / 90} \geq 8 / 256 \mu \mathrm{g} / \mathrm{mL}\right)$ but also remained highly susceptible to carbapenems $\left(\mathrm{MIC}_{50 / 90} \leq 0.125 / 0.25 \mu \mathrm{g} / \mathrm{mL}\right)$ and flomoxef $\left(0.06 / 0.25 \mu \mathrm{g} / \mathrm{mL} \leq \mathrm{MIC}_{50 / 90} \leq 1 / 16 \mu \mathrm{g} / \mathrm{mL}\right)$, as revealed in this study. Similarly, cephamycins remained largely effective against ESBL-producing strains [19,21]. However, ESBL producers in the reported studies showed greater susceptibility to $\beta$-lactam combinations, such as amoxicillin-clavulanate $\left(\mathrm{MIC}_{50 / 90}=8 / 16 \mu \mathrm{g} / \mathrm{mL}\right)$ and piperacillintazobactam $\left(2 / 64 \mu \mathrm{g} / \mathrm{mL} \leq \mathrm{MIC}_{50 / 90} \leq 8 / 128 \mu \mathrm{g} / \mathrm{mL}\right)$, compared to the strains examined in this study. Although piperacillin-tazobactam and cefepime have shown comparable efficacy as carbapenems for infections caused by ESBL producers, this use is only recommended when the organisms are tested susceptible to these antibiotics [26]. The highly resistant phenotype of the local ESBL producers seen in this study suggested against the use of piperacillin-tazobactam and cefepime as potential carbapenem-sparing strategies in Malaysia. Cephamycins may be an alternative to carbapenems for the treatment of UTIs caused by ESBL producers [26]. Based on the data obtained in this study, cefmetazole would be a better option compared to cefoxitin, since a greater susceptibility to cefmetazole was seen with the ESBL-producing strains.

A comparison of the $\mathrm{MIC}_{50 / 90}$ values obtained in this study showed that flomoxef exhibited a higher in vitro efficacy against ESBL-producing E. coli and K. pneumoniae than most of the $\beta$-lactam antibiotics tested, except for carbapenems. In the present study, a reduced susceptibility to flomoxef was found associated with an increased resistance to cefmetazole, while carbapenems remained consistently effective against all bacterial strains. Our results showed that carbapenems had the highest activity against the ESBL-producing strains, with the lowest $\mathrm{MIC}_{50 / 90}$ values $(\leq 0.03 / 0.06-1 / 1 \mu \mathrm{g} / \mathrm{mL})$. On the other hand, the $\mathrm{MIC}_{50 / 90}$ of flomoxef were lower than cefmetazole and cefoxitin in both E. coli (twoto sixteen-fold reductions) and K. pneumoniae (two- to four-fold reductions), indicating a slightly higher in vitro efficacy of flomoxef against the ESBL producers. Consistent observations were made in studies conducted in China, Japan, and Korea, where ESBL producers isolated in these regions were most susceptible to carbapenems, followed by flomoxef and cephamycins (cefmetazole or cefoxitin) $[17,19,21]$. Clinical studies investigating the use of flomoxef for the treatment of UTIs caused by ESBL-producing E. coli showed promising results and comparable efficacy with carbapenems [22,23]. However, a more variable outcome was produced when used in the treatment of bloodstream infections caused by ESBL producers [26]. Taiwanese studies have documented increased mortality with the use of flomoxef in hemodialysis access-related bacteremia and lower efficacy compared to carbapenems for isolates with flomoxef MIC of $2-8 \mu \mathrm{g} / \mathrm{mL}[27,28]$. Based on the MIC data obtained in this study, flomoxef may be considered as a potential alternative to carbapenems for less severe infections, such as UTIs caused by ESBL-producing E. coli and $K$. pneumoniae in this region.

Most of the UTI-associated E. coli and K. pneumoniae strains examined in this study carried at least one gene encoding for class A $\beta$-lactamases, which are known to confer a resistance to penicillins, extended-spectrum cephalosporins, and monobactams [29]. The majority of the strains carried two to three ESBL genes, likely accounting for the high MIC values of $\beta$-lactam antibiotics observed in this study. Moreover, similar ESBL gene combinations could have explained the common $\beta$-lactam-resistant phenotypes shared between the E. coli and K. pneumoniae strains. Our findings are in agreement with local 
studies that identified $b l a_{\mathrm{CTX}-\mathrm{M}}$ as the most prevalent gene among the ESBL-producing E. coli and K. pneumoniae isolated from human and animal sources [30-33]. In these local reports, ESBL producers often carried multiple ESBL genes conferring a wide range of $\beta$-lactam resistance in the organisms, which is consistently observed in this study. The absence of $b l a_{\mathrm{FOX}}$ within our strains pool is probably not unexpected, given that the rates of resistance to cephamycins (cefmetazole and cefoxitin) were relatively low among $E$. coli and K. pneumoniae. Consistent with the previous report, the cephamycins remained active against the majority of the investigated strains, as these organisms mostly produced TEMand/or SHV-type $\beta$-lactamases that are ineffective against cephamycins [34]. Approximately one-fifth of the ESBL-producing E. coli did not harbor any of the four ESBL genes that were examined in this study. Since porin- and efflux-mediated mechanisms are not common in E. coli and often only confer resistance to antibiotics at low concentrations, other ESBL genes $\left(b l a_{\mathrm{OXA}}, b l a_{\mathrm{PER}}, b l a_{\mathrm{GES}}\right.$, etc.) could be responsible for the ESBL-producing phenotype of these strains $[35,36]$. Moreover, the production of AmpC $\beta$-lactamases, especially among E. coli, is frequently associated with positive ESBL test outcomes [37,38].

One of the limitations of this study was the low number of ESBL-producing E. coli and K. pneumoniae collected within one year from a single study center in Malaysia. Although our strain pools may not represent the entire ESBL-producing Enterobacteriaceae population in this region, both temporally and geographically, the data generated in this study provided an insight into the extent of $\beta$-lactam resistance among ESBL producers associated with UTIs in Malaysia. Moreover, the susceptibility data obtained in this study support further consideration on the potential use of flomoxef as an alternative treatment for UTIs caused by ESBL producers. Another limitation of the study was that only four $\beta$-lactamase genes were targeted; therefore, the data obtained did not provide a comprehensive molecular epidemiology of ESBL production among the UTI-associated E. coli and K. pneumoniae strains in this region.

\section{Materials and Methods}

\subsection{Study Site and Patient Data Collection}

Bacterial strains collection was conducted at the University Malaya Medical Centre (UMMC). UMMC is a tertiary hospital with a total of 1623 beds, located at the capital city Kuala Lumpur in Malaysia. Basic demographic data of patients were retrieved from the Patient Information Department (previously known as the Medical Record Unit) of UMMC.

\subsection{Bacterial Strains Collection}

A total of 100 ESBL producers, comprising of 50 E. coli and 50 K. pneumoniae strains, were obtained from the UMMC diagnostic laboratory's strain collections. All strains were isolated from urine samples of patients admitted to UMMC in 2015 (January-November). The isolation and identification of the bacterial strains, along with the detection of ESBL production, were part of the routine microbiological examination procedures in the hospital's diagnostic laboratory. Only ESBL-producing E. coli and K. pneumoniae were selected for this study. Other bacterial species and nonproducers of ESBL isolated from urine samples were excluded from the study. All selected ESBL-producing strains $(n=100)$ were confirmed as the first isolate from urine samples, and only one strain was collected per patient. Bacterial strain collections were terminated upon reaching the targeted number of strains (50 strains for each species). E. coli and K. pneumoniae strains were further confirmed by Polymerase Chain Reaction (PCR) through the detection of $p h o A$ and $m d h$ genes, respectively $[39,40]$. AMR data of the bacterial strains pertaining to commonly prescribed antibiotics for UTIs (cefuroxime, ciprofloxacin, nitrofurantoin, and trimethoprim-sulfamethoxazole) were retrieved from the diagnostic laboratory's database. These four antibiotics were not included in the broth microdilution assays performed in this study. 


\subsection{Broth Microdilution Assays}

The antimicrobial susceptibility of the E. coli and K. pneumoniae strains to $\beta$-lactam antibiotics, including flomoxef, was determined. MIC of penicillins (ampicillin, penicillin$\mathrm{G}$, piperacillin, and ticarcillin); $\beta$-lactam combination agents (amoxicillin-clavulanate, ceftazidime-clavulanate, cefotaxime-clavulanate, ticarcillin-clavulanate, and piperacillintazobactam); cephems (cefmetazole, cefoxitin, ceftazidime, cefoperazone, ceftriaxone, cefotaxime, and cefepime); carbapenems (imipenem and meropenem); and flomoxef were determined using the broth microdilution method. The MIC values were interpreted according to the breakpoints recommended by the CLSI guidelines [41]. ESBL-producing phenotype of the bacterial strains was confirmed by at least 8-fold reduction in the MIC of ceftazidime with $4-\mu \mathrm{g} / \mathrm{mL}$ clavulanate (fixed dose) when compared to the MIC of ceftazidime alone or cefotaxime-clavulanate $(4-\mu \mathrm{g} / \mathrm{mL}$ fixed dose) when compared to cefotaxime [41]. CLSI-recommended threshold for flomoxef was not available; therefore, the MIC breakpoints for moxalactam (an oxacephem antibiotic) were used to predict the bacterial susceptibility to flomoxef [21]. Scatter plots were constructed to illustrate the relative efficacy of flomoxef in comparison with the $\beta$-lactam antibiotics that remained active against ESBL-producing E. coli and K. pneumoniae strains in this study. The $\log _{10}$ MIC values of the comparator antibiotics were plotted against that of flomoxef.

\subsection{Detection of $\beta$-Lactamase-Encoding Genes}

PCR was performed to detect the presence of ESBL genes $\left(b l a_{\mathrm{CTX}-\mathrm{M}}, b l a_{\mathrm{SHV}}\right.$, and $\left.b l a_{\mathrm{TEM}}\right)$ and the plasmid-borne AmpC-type $\beta$-lactamase gene $\left(b l a_{\mathrm{FOX}}\right)$ in the bacterial strains. Primer sequences, PCR reaction mixture, and thermocycling conditions were adapted from published studies $[42,43]$. The annealing temperatures of the primers were optimized at $55^{\circ} \mathrm{C}\left(b l a_{\mathrm{CTX}-\mathrm{M}}\right), 58^{\circ} \mathrm{C}\left(\right.$ bla $a_{\mathrm{SHV}}$ and $\left.b l a_{\mathrm{TEM}}\right)$, and $60{ }^{\circ} \mathrm{C}\left(b l a_{\mathrm{FOX}}\right)$.

\section{Conclusions}

In this study, ESBL-producing E. coli and K. pneumoniae associated with UTIs in Malaysia were found to be highly resistant to penicillins, third- and fourth-generation cephalosporins, and $\beta$-lactam combination agents. Carbapenems showed the highest in vitro efficacy to ESBL-producing strains, followed by flomoxef. Therefore, due to the low prevalence of resistance to flomoxef detected here, this antibiotic can be considered as an alternative to carbapenems in clinical practice in Malaysia for the treatment of UTIs caused by ESBL-producing E. coli and K. pneumoniae. We recommend the continuous surveillance of ESBL producers at both the institutional and national levels to preserve the clinical efficacy of broad-spectrum cephalosporins and prevent the emergence of carbapenem resistance in local Enterobacteriaceae strains.

Author Contributions: Conceptualization, C.S.J.T., L.H.T., K.C.L. and S.A.; methodology, C.S.J.T. and S.A.; software, C.W.C.; validation, S.T.N., K.A.J., S.C.T. and L.H.Y.; formal analysis, S.T.N. and C.W.C.; investigation, S.T.N., K.A.J., S.C.T. and L.H.Y.; resources, C.S.J.T., S.A., L.H.T. and K.C.L.; data curation, S.T.N., C.W.C., K.A.J., S.C.T. and L.H.Y.; writing-original draft preparation, S.T.N.; writing-review and editing, C.S.J.T., S.A., C.W.C., K.A.J., K.C.L., S.C.T. and L.H.T.; visualization, S.T.N., C.W.C., K.A.J., S.C.T. and L.H.Y.; supervision, C.S.J.T. and S.A.; project administration, S.T.N.; funding acquisition, S.A., L.H.T. and K.C.L. All authors have read and agreed to the published version of the manuscript.

Funding: This research was funded by the University of Malaya Research Fund, grant number RU008-2018 and Shionogi Singapore Pte. Ltd.

Institutional Review Board Statement: The study was conducted according to the guidelines of the Declaration of Helsinki and approved by the Medical Ethics Committee of the University Malaya Medical Centre (UMMC) (protocol code MEC-ID: 1073.21 on 6 June 2014).

Data Availability Statement: All data presented in this study are available in this published article. 
Acknowledgments: This was a collaborative study between the University of Malaya (UM) and Shionogi Singapore Pte. Ltd. We thank both UM and Shionogi for providing the materials and facilities for this study.

Conflicts of Interest: The authors declare no conflict of interest.

\section{References}

1. Foxman, B. Urinary Tract Infection. In Women and Health, 2nd ed.; Goldman, M.B., Troisi, R., Rexrode, K.M., Eds.; Academic Press: Cambridge, MA, USA, 2013; pp. 553-564.

2. Foxman, B. Urinary tract infection syndromes: Occurrence, recurrence, bacteriology, risk factors, and disease burden. Infect. Dis Clin. N. Am. 2014, 28, 1-13. [CrossRef]

3. Gupta, K.; Hooton, T.M.; Naber, K.G.; Wullt, B.; Colgan, R.; Miller, L.G.; Moran, G.J.; Nicolle, L.E.; Raz, R.; Schaeffer, A.J.; et al. International clinical practice guidelines for the treatment of acute uncomplicated cystitis and pyelonephritis in women: A 2010 update by the Infectious Diseases Society of America and the European Society for Microbiology and Infectious Diseases. Clin. Infect. Dis. 2011, 52, e103-e120. [CrossRef] [PubMed]

4. Ministry of Health (MOH) Malaysia. National Antimicrobial Guideline 2019; Pharmaceutical Services Programme, MOH: Petaling Jaya, Malaysia, 2019.

5. Ronald, A. The etiology of urinary tract infection: Traditional and emerging pathogens. Am. J. Med. 2002, 113, 14-19. [CrossRef]

6. Flores-Mireles, A.L.; Walker, J.N.; Caparon, M.G.; Hultgren, S.J. Urinary tract infections: Epidemiology, mechanisms of infection and treatment options. Nat. Rev. Genet. 2015, 13, 269-284. [CrossRef] [PubMed]

7. McLellan, L.K.; Hunstad, D.A. Urinary Tract Infection: Pathogenesis and Outlook. Trends Mol. Med. 2016, 22, 946-957. [CrossRef] [PubMed]

8. Chu, C.M.; Lowder, J.L. Diagnosis and treatment of urinary tract infections across age groups. Am. J. Obstet. Gynecol. 2018, 219, 40-51. [CrossRef] [PubMed]

9. Doi, Y.; Iovleva, A.; Bonomo, R.A. The ecology of extended-spectrum $\beta$-lactamases (ESBLs) in the developed world. J. Travel Med. 2017, 24, S44-S51. [CrossRef]

10. National Surveillance of Antimicrobial Resistance, Malaysia. Institute of Medical Research, Kuala Lumpur. Available online: https://www.imr.gov.my/MyOHAR/index.php/site/archive_rpt (accessed on 22 September 2020).

11. Fazlul, M.K.K.; Rashid, S.S.; Nazmul, M.H.M.; Zaidul, I.S.M.; Baharudin, R.; Ramli, A.N.M. A clinical update on antibiotic resistance Gram-negative bacteria in Malaysia-A review. J. Int. Pharm. Res. 2019, 45, 270-283.

12. Kawamoto, I.; Miyauchi, M. $\beta$-Lactams and Other Antimicrobial Agents. In Antibiotics; Kawamoto, I., Ed.; CRC Press: Boca Raton, FL, USA, 1992.

13. Sato, H.; Narita, A.; Suzuki, H.; Mastumoto, K.; Nakanishi, Y.; Nakazawa, S.; Niino, K.; Nakada, Y. The study of flomoxef in the pediatric field. Jpn. J. Antibiot. 1987, 40, 1349-1363.

14. Yokoyama, T.; Kodama, T.; Takesue, Y.; Fujimoto, M.; Hiyama, E.; Ichikawa, T. Studies on antibacterial activity of flomoxef and its distribution to serum and intraperitoneal exudate in surgery. Jpn. J. Antibiot. 1987, 40, 1809-1819.

15. Kanegae, H.; Yamada, H.; Yamaguchi, T.; Kuroki, S.; Katoh, O. Clinical studies on flomoxef in respiratory tract infections. Jpn. J. Antibiot. 1987, 40, 1803-1808.

16. Matsumura, Y.; Yamamoto, M.; Nagao, M.; Komori, T.; Fujita, N.; Hayashi, A.; Shimizu, T.; Watanabe, H.; Doi, S.; Tanaka, M.; et al. Multicenter retrospective study of cefmetazole and flomoxef for treatment of extended-spectrum- $\beta$-lactamase-producing Escherichia coli bacteremia. Antimicrob. Agents Chemother. 2015, 59, 5107-5113. [CrossRef]

17. Miyazaki, M.; Yamada, Y.; Matsuo, K.; Komiya, Y.; Uchiyama, M.; Nagata, N.; Takata, T.; Jimi, S.; Imakyure, O. Change in the antimicrobial resistance profile of extended-spectrum $\beta$-lactamase-producing Escherichia coli. J. Clin. Med. Res. 2019, 11, 635-641. [CrossRef] [PubMed]

18. Takesue, Y.; Kusachi, S.; Mikamo, H.; Sato, J.; Watanabe, A.; Kiyota, H.; Iwata, S.; Kaku, M.; Hanaki, H.; Sumiyama, S.; et al. Antimicrobial susceptibility of common pathogens isolated from postoperative intra-abdominal infections in Japan. J. Infect. Chemother. 2018, 24, 330-340. [CrossRef]

19. Jung, Y.; Lee, S.S.; Song, W.; Kim, H.-S.; Uh, Y. In vitro activity of flomoxef against extended-spectrum $\beta$-lactamase-producing Escherichia coli and Klebsiella pneumoniae in Korea. Diagn. Microbiol. Infect. Dis. 2019, 94, 88-92. [CrossRef] [PubMed]

20. Lee, C.-H.; Su, L.-H.; Tang, Y.-F.; Liu, J.-W. Treatment of ESBL-producing Klebsiella pneumoniae bacteraemia with carbapenems or flomoxef: A retrospective study and laboratory analysis of the isolates. J. Antimicrob. Chemother. 2006, 58, 1074-1077. [CrossRef] [PubMed]

21. Yang, Q.; Zhang, H.; Cheng, J.; Xu, Z.; Xu, Y.-C.; Cao, B.; Kong, H.; Ni, Y.; Yu, Y.; Sun, Z.; et al. In vitro activity of flomoxef and comparators against Escherichia coli, Klebsiella pneumoniae and Proteus mirabilis producing extended-spectrum $\beta$-lactamases in China. Int. J. Antimicrob. Agents 2015, 45, 485-490. [CrossRef]

22. Horie, A.; Nariai, A.; Katou, F.; Abe, Y.; Saito, Y.; Koike, D.; Hirade, T.; Ito, T.; Wakuri, M.; Fukuma, A. Increased communityacquired upper urinary tract infections caused by extended-spectrum beta-lactamase-producing Escherichia coli in children and the efficacy of flomoxef and cefmetazole. Clin. Exp. Nephrol. 2019, 23, 1306-1314. [CrossRef] 
23. Abe, Y.; Inan-Erdogan, I.; Fukuchi, K.; Wakabayashi, H.; Ogawa, Y.; Hibino, S.; Sakurai, S.; Matsuhashi, K.; Watanabe, Y.; Hashimoto, K.; et al. Efficacy of non-carbapenem antibiotics for pediatric patients with first febrile urinary tract infection due to extended-spectrum beta-lactamase-producing Escherichia coli. J. Infect. Chemother. 2017, 23, 517-522. [CrossRef] [PubMed]

24. Teng, C.L.; Tong, S.-F.; Khoo, E.; Lee, V.; Zailinawati, A.H.; Mimi, O.; Chen, W.S.; Nordin, S. Antibiotics for URTI and UTIprescribing in Malaysian primary care settings. Aust. Fam. Physician 2011, 40, 325-329. [PubMed]

25. Ab Rahman, N.; Teng, C.L.; Sivasampu, S. Antibiotic prescribing in public and private practice: A cross-sectional study in primary care clinics in Malaysia. BMC Infect. Dis. 2016, 16, 208. [CrossRef] [PubMed]

26. Karaiskos, I.; Giamarellou, H. Carbapenem-sparing strategies for ESBL producers: When and how. Antibiotics 2020, 9, 61. [CrossRef]

27. Lee, C.H.; Su, L.H.; Chen, F.J.; Tang, Y.F.; Li, C.C.; Chien, C.C.; Liu, J.W. Comparative effectiveness of flomoxef versus car-bapenems in the treatment of bacteraemia due to extended-spectrum $\beta$-lactamase-producing Escherichia coli or Klebsiella pneumoniae with emphasis on minimum inhibitory concentration of flomoxef: A retrospective study. Int. J. Antimicrob. Agents 2015, 46, 610-615.

28. Yang, C.C.; Li, S.H.; Chuang, F.R.; Chen, C.H.; Lee, C.H.; Chen, J.B.; Wu, C.H.; Lee, C.T. Discrepancy between effects of carbapenems and flomoxef in treating nosocomial hemodialysis access-related bacteremia secondary to extended spectrum betalactamase producing Klebsiella pneumoniae in patients on maintenance hemodialysis. BMC Infect. Dis. 2012, 12, 206. [CrossRef] [PubMed]

29. Bush, K.; Jacoby, G.A. Updated functional classification of beta-lactamases. Antimicrob. Agents Chemother. 2010, 54, 969-976. [CrossRef] [PubMed]

30. Mohsen, S.M.Y.; Hamzah, H.A.; Al-Deen, M.M.I.; Baharudin, R. Antimicrobial susceptibility of Klebsiella pneumoniae and Escherichia coli with extended-spectrum $\beta$-lactamase associated genes in Hospital Tengku Ampuan Afzan, Kuantan, Pahang. Malays. J. Med Sci. 2016, 23, 14-20.

31. Ho, W.S.; Balan, G.; Puthucheary, S.; Kong, B.H.; Lim, K.T.; Tan, L.K.; Koh, X.P.; Yeo, C.C.; Thong, K.L. Prevalence and characterization of multi-drug-resistant and extended-spectrum beta-lactamase-producing Escherichia coli from pediatric wards of a Malaysian hospital. Microb. Drug Resist. 2012, 18, 408-416. [CrossRef]

32. Mobasseri, G.; Thong, K.L.; Rajasekaram, G.; Teh, C.S.J. Molecular characterization of extended-spectrum $\beta$-lactamase-producing Klebsiella pneumoniae from a Malaysian hospital. Braz. J. Microbiol. 2019, 51, 189-195. [CrossRef]

33. Mobasseri, G.; Teh, C.S.J.; Toung, O.P.; Thong, K.L. The emergence of colistin-resistant Klebsiella pneumoniae strains from swine in Malaysia. J. Glob. Antimicrob. Resist. 2019, 17, 227-232. [CrossRef]

34. Jacoby, G.A.; Medeiros, A.A. More extended-spectrum beta-lactamases. Antimicrob. Agents Chemother. 1991, 35, 1697-1704. [CrossRef]

35. Shaikh, S.; Fatima, J.; Shakil, S.; Rizvi, S.M.D.; Kamal, M.A. Antibiotic resistance and extended spectrum beta-lactamases: Types, epidemiology and treatment. Saudi J. Biol. Sci. 2015, 22, 90-101. [CrossRef]

36. Cag, Y.; Caskurlu, H.; Fan, Y.; Cao, B.; Vahaboglu, H. Resistance mechanisms. Ann. Transl. Med. 2016, 4, 326. [CrossRef]

37. Munier, G.K.; Johnson, C.L.; Snyder, J.W.; Moland, E.S.; Hanson, N.D.; Thomson, K.S. Positive extended-spectrum- $\beta$-lactamase (ESBL) screening results may be due to ampC $\beta$-lactamases more often than to ESBLs. J. Clin. Microbiol. 2010, 48, 673-674. [CrossRef] [PubMed]

38. Robberts, F.J.L.; Kohner, P.C.; Patel, R. Unreliable extended-spectrum $\beta$-lactamase detection in the presence of plasmid-mediated AmpC in Escherichia coli clinical isolates. J. Clin. Microbiol. 2009, 47, 358-361. [CrossRef] [PubMed]

39. Diancourt, L.; Passet, V.; Verhoef, J.; Grimont, P.A.D.; Brisse, S. Multilocus sequence typing of Klebsiella pneumoniae nosocomial isolates. J. Clin. Microbiol. 2005, 43, 4178-4182. [CrossRef] [PubMed]

40. Kong, R.Y.C.; So, C.L.; Law, W.F.; Wu, R.S.S. A sensitive and versatile multiplex PCR system for the rapid detection of enterotoxigenic (ETEC), enterohaemorrhagic (EHEC) and enteropathogenic (EPEC) strains of Escherichia coli. Mar. Pollut. Bull. 1999, 38, 1207-1215. [CrossRef]

41. Clinical and Laboratory Standards Institute (CLSI). Performance Standards for Antimicrobial Susceptibility Testing, 30th ed.; CLSI Suppl. M100; Clinical and Laboratory Standards Institute (CLSI): Wayne, PA, USA, 2020.

42. Dallenne, C.; Da Costa, A.; Decré, D.; Favier, C.; Arlet, G. Development of a set of multiplex PCR assays for the detection of genes encoding important $\beta$-lactamases in Enterobacteriaceae. J. Antimicrob. Chemother. 2010, 65, 490-495. [CrossRef]

43. Eckert, C.; Gautier, V.; Saladin-Allard, M.; Hidri, N.; Verdet, C.; Ouldhocine, Z.; Barnaud, G.; Delisle, F.; Rossier, A.; Lambert, T.; et al. Dissemination of CTX-M-Type $\beta$-Lactamases among clinical isolates of Enterobacteriaceae in Paris, France. Antimicrob. Agents Chemother. 2004, 48, 1249-1255. [CrossRef] 